\title{
FLAVONOIDES DE Lonchocarpus campestris (Leguminosae)
}

\author{
Andreza Maria L. Pires, Edilberto R. Silveira e Otília Deusdênia L. Pessoa* \\ Departamento de Química Orgânica e Inorgânica, Centro de Ciências, Universidade Federal do Ceará, CP 12200, 60021-970 \\ Fortaleza - CE, Brasil
}

Recebido em 16/3/10; aceito em 13/9/10; publicado na web em 8/12/10

\begin{abstract}
FLAVONOIDS FROM Lonchocarpus campestris (Leguminosae). A new flavone named 3,4',5,6-tetramethoxy-[2", 3":7,8] furanoflavone besides the known flavonoids $(2 S, 3 R, 4 S)$-3,4,5,8-tetramethoxy-[2",3":6,7]-furanoflavan, 3,6-dimethoxy2",2"-dimethylcromene-[2",3":7,8]-flavone, 3,5,6-trimethoxy-[2",3":7,8]-furanoflavone, 2,4",4,5-tetramethoxy-[2",3":6,7]furanodihydroaurone, $(2 R, 3 S, 4 S)$-3,4,5,6-tetramethoxy-[2",3"'7,8]-furanoflavan and 3',4'-methylenodioxy-5,6-dimethoxy[2",3":7,8]-furanoflavone were isolated from the root barks of Lonchocarpus campestris. The complete ${ }^{1} \mathrm{H}$ and ${ }^{13} \mathrm{C}$ NMR assignments of the new furan flavonoid was performed using $1 \mathrm{D}$ and 2D pulse sequences, including COSY, HMQC and HMBC experiments.
\end{abstract}

Keywords: Lonchocarpus campestris; Leguminosae; furanoflavonoids.

\section{INTRODUÇÃO}

A família Leguminosae (Fabaceae) constitui a terceira maior família botânica, com aproximadamente 18.000 espécies distribuídas em 619 gêneros, boa parte pertencendo originalmente à flora brasileira. ${ }^{1,2}$

Lonchocarpus, um dos gêneros desta grande família, é representado por cerca de 100 espécies, predominando nas regiões tropicais e subtropicais. Para a flora brasileira, são reconhecidas cerca de 23 espécies. ${ }^{3}$ Estudos fitoquímicos mostram que as plantas que compõem este gênero são produtoras, em potencial, de compostos fenólicos, representados especialmente por flavonas, chalconas, cromonas, flavanas, flavanonas e auronas. ${ }^{3-6}$ Em geral, os flavonoides produzidos por Lonchocarpus são de baixa polaridade e uma particularidade destes compostos é a presença frequente de anéis furânicos ou pirânicos, os quais podem estar ligados ao núcleo flavonoídico em C-6/C-7 ou C-7/C-8, formando sistemas lineares ou angulares, respectivamente. Grupos metoxilas e prenilas são outros substituintes frequentes.

Uma série de atividades, tais como antimicrobiana, ${ }^{5}$ gastroprotetora, ${ }^{7}$ citotóxica, ${ }^{8}$ antiagregante plaquetária ${ }^{9}$ e antimalárica, ${ }^{10}$ foram relatadas para flavonoides isolados de exemplares do gênero Lonchocarpus.

Neste trabalho são apresentados os resultados obtidos com a investigação fitoquímica de Lonchocarpus campestris.

\section{PARTE EXPERIMENTAL}

\section{Procedimentos experimentais gerais}

Os pontos de fusão foram obtidos em equipamento de microdeterminação digital da Mettler Toledo com placa aquecedora FP82HT e central de processamento FP90. As determinações foram realizadas a uma velocidade de aquecimento de $2{ }^{\circ} \mathrm{C} / \mathrm{min}$ e não foram corrigidas. Os espectros no infravermelho foram registrados em espectrômetro Perkin-Elmer Spectrum 1000 FTIR, utilizando pastilhas de $\mathrm{KBr}$. Os espectros de $\mathrm{RMN}{ }^{1} \mathrm{He}{ }^{13} \mathrm{C}$, uni- e bidimensio-

*e-mail: opessoa@ufc.br nais, foram obtidos em um espectrômetro Bruker, modelo Avance DRX-500 (500 MHz para ${ }^{1} \mathrm{H}$ e $125 \mathrm{MHz}$ para ${ }^{13} \mathrm{C}$ ). Os espectros de massas foram obtidos em espectrômetro Shimadzu, modelo QP 5000/DI-50, por impacto eletrônico de 70 eV. Nas cromatografias de adsorção utilizou-se gel de sílica 60 da Vetec $(\varnothing \mu \mathrm{m}$ 70-230 mesh, para cromatografias em colunas) e Merck ( $\varnothing \mu \mathrm{m}$ 230-400 mesh, para cromatografias com média pressão, flash), enquanto as cromatografias em camada delgada analítica (CCDA) foram realizadas em cromatofolha de alumínio (Merck) com indicador de fluorescência $\left(\mathrm{F}_{254}\right)$. As substâncias foram reveladas por aspersão de solução de vanilina/ácido perclórico/EtOH, seguida de aquecimento em estufa $\left(\approx 100^{\circ} \mathrm{C}\right)$.

\section{Material vegetal}

As raízes de Lonchocarpus campestris foram coletadas em abril de 2006, na localidade de Alcântaras, situada na Serra da Meruoca, Ceará. A identificação da planta, cuja exsicata (No. 39175) se encontra depositada no Herbário Prisco Bezerra - UFC, foi realizada pelo Prof. A. G. Fernandes, do Departamento de Biologia da Universidade Federal do Ceará.

\section{Extração e isolamento}

As cascas das raízes $(900 \mathrm{~g})$, secas à temperatura ambiente e trituradas, foram submetidas à percolação com hexano e posteriormente com etanol. Os extratos foram filtrados e destilados sob pressão reduzida a aproximadamente $50{ }^{\circ} \mathrm{C}$, fornecendo $19,4 \mathrm{~g}$ de extrato hexânico e 60,3 g de extrato etanólico.

O extrato hexânico $(19,4 \mathrm{~g})$ foi fracionado em coluna de gel de sílica empregando hexano, hexano/ $\mathrm{CH}_{2} \mathrm{Cl}_{2} 1: 1, \mathrm{CH}_{2} \mathrm{Cl}_{2}$, AcOEt e $\mathrm{MeOH}$ como eluentes. Durante a evaporação dos solventes das frações hexano/ $\mathrm{CH}_{2} \mathrm{Cl}_{2}$ 1:1, $\mathrm{CH}_{2} \mathrm{Cl}_{2}$ e AcOEt houve a formação de precipitados, os quais foram separadamente filtrados e purificados por recristalização. Da fração hexano/ $\mathrm{CH}_{2} \mathrm{Cl}_{2} 1: 1$ (4,6 g), isolouse por recristalização em hexano/AcOEt 8:2, o composto 1 (447 mg). Da fração $\mathrm{CH}_{2} \mathrm{Cl}_{2}(4,3 \mathrm{~g})$, após recristalização em AcOEt, obteve-se o composto 2 (156,6 mg), enquanto da fração AcOEt 
(2,8 g) isolou-se por recristalização em AcOEt, a substância 3 (150 $\mathrm{mg}$ ). O líquido mãe da fração $\mathrm{CH}_{2} \mathrm{Cl}_{2}$ foi concentrado $(4,0 \mathrm{~g}) \mathrm{e}$ submetido à cromatografia em coluna gravitacional sobre gel de sílica, usando como eluentes hexano/ $\mathrm{CH}_{2} \mathrm{Cl}_{2}$ 6:4, 4:6, 2:8, $\mathrm{CH}_{2} \mathrm{Cl}_{2}$, AcOEt e $\mathrm{MeOH}$. Um total de 106 frações de aproximadamente $10 \mathrm{~mL}$ foram coletadas, as quais, após monitoramento por CCD, resultaram em 12 frações. A fração 18-45 (1,56 g), obtida por eluição com hexano/ $\mathrm{CH}_{2} \mathrm{Cl}_{2} 6: 4$, foi submetida à cromatografia sobre média pressão (flash), empregando como eluente, hexano/ AcOEt 8:2, para fornecer o composto $4(368,2 \mathrm{mg})$. O liquido mãe da fração AcOEt $(2,2 \mathrm{~g})$ foi submetido à cromatografia em coluna sobre gel de sílica, empregando como eluente $\mathrm{CH}_{2} \mathrm{Cl}_{2}, \mathrm{CH}_{2} \mathrm{Cl}_{2} /$ AcOEt 9:1, 7:3, 1:1, 3:7, 1:9, seguido de $\mathrm{MeOH}$. Ao final deste procedimento, foram coletadas 37 frações de $20 \mathrm{~mL}$, as quais, após monitoramento em $\mathrm{CCD}$, foram reunidas em 11 frações. A fração $\mathrm{F} 8 / 20$ (1,35 g), resultado da eluição com $\mathrm{CH}_{2} \mathrm{Cl}_{2}$ e $\mathrm{CH}_{2} \mathrm{Cl}_{2} / \mathrm{AcOEt}$ 9:1, foi submetida a sucessivas cromatografias flash empregando para a eluição uma mistura ternária de hexano/ $\mathrm{CH}_{2} \mathrm{Cl}_{2} /$ acetona $(6,8: 2,6: 0,6)$ para fornecer $5(34,2 \mathrm{mg})$

$\mathrm{O}$ extrato $\mathrm{EtOH}(60,3 \mathrm{~g})$ foi fracionado sobre gel de sílica obtendo-se ao final do procedimento as frações: $\mathrm{CH}_{2} \mathrm{Cl}_{2}(10,6$ $\mathrm{g})$, AcOEt $(26,5 \mathrm{~g})$ e $\mathrm{MeOH}(5,8 \mathrm{~g})$. A fração $\mathrm{CH}_{2} \mathrm{Cl}_{2}(10,6)$ foi submetida à cromatografia em coluna de gel sílica utilizando para eluição hexano/AcOEt 7:3, 6:4, 1:1, 3:7, 2:8, AcOEt e MeOH. Foram obtidas 42 frações de $30 \mathrm{~mL}$. Após comparação por CCD, as frações 5-6 (1,45 g), obtidas por eluição com hexano/AcOEt $7: 3$, foram reunidas e submetidas a consecutivas cromatografias flash empregando $\mathrm{CH}_{2} \mathrm{Cl}_{2}$ e hexano/ $\mathrm{CH}_{2} \mathrm{Cl}_{2}$ 2:8, respectivamente, para fornecer o composto $6(23 \mathrm{mg})$. A fração AcOEt (26,0 g) foi submetida à cromatografia de adsorção em coluna de gel sílica utilizando para a eluição $\mathrm{CH}_{2} \mathrm{Cl}_{2}, \mathrm{CH}_{2} \mathrm{Cl}_{2} / \mathrm{AcOEt} 1: 1$, AcOEt e $\mathrm{MeOH}$, fornecendo 45 frações de $30 \mathrm{~mL}$. As frações 3-16, obtidas por eluição com $\mathrm{CH}_{2} \mathrm{Cl}_{2}$, após análise em $\mathrm{CCD}$, foram reunidas, rendendo $1,44 \mathrm{~g}$ de material, o qual foi submetido à cromatografia em coluna de sílica gel utilizando a seguinte série de eluentes: hexano/AcOEt 7:3, 6:4, 2:8, AcOEt e MeOH. Foram coletadas 37 frações de $20 \mathrm{~mL}$. As frações 11-14 (446 mg), obtidas por eluição com hexano/AcOEt 7:3, após análise em CCD, foram reunidas e durante o processo de evaporação do solvente houve a formação de um precipitado, o qual foi separado do líquido mãe por filtração e posteriormente lavado com a mistura hexano/AcOEt 8:2, obtendo-se assim o composto 7 (13 mg).

(2S,3R,4S)-3, 4, 5, 8-Tetrametoxi-[2", 3": 6, 7]-furanoflava$n a$ (1). Cristais incolores. p.f. $110,9-111,7{ }^{\circ} \mathrm{C},[\alpha]_{\mathrm{D}}^{20}-9^{\circ}(0,004$, $\left.\mathrm{CHCl}_{3}\right)$, IV (KBr), cm${ }^{-1}: 2934 ; 2829 ; 1623 ; 1544 ; 1485 ; 1411 ; 1350$; 1252; 1190; 1137; 1096. EM (Int. rel.) $\mathrm{m} / z: 370$ ([M] $\left.{ }^{+}, 100\right), 339$ (22), 236 (87), 221 (60), 193 (37), 134 (15), 121 (50), 77 (16). Os dados de $\mathrm{RMN}{ }^{13} \mathrm{Ce} \mathrm{e}^{1} \mathrm{H}\left(\mathrm{CDCl}_{3}\right)^{3}$ estão descritos nas Tabelas 1 e 2, respectivamente.

3,6-Dimetoxi-2", 2"-dimetilcromeno-[2", 3": 7, 8]-flavona (2). Cristais incolores. p.f. $197,1-199,0{ }^{\circ} \mathrm{C}$, IV (KBr), $\mathrm{cm}^{-1}$ : 1625; 1470; 1397; 1287; 1217; 1168; 1116; 1069. EM (Int. rel.) $\mathrm{m} / \mathrm{z}: 364$ ([M] $]^{+}$, 100), 349 (85), 319 (6) 249 (6), 217 (14), 174 (30), 105 (12), 77 (15). Os dados de RMN ${ }^{13} \mathrm{C} \mathrm{e}{ }^{1} \mathrm{H}\left(\mathrm{CDCl}_{3}\right)^{11}$ estão descritos nas Tabelas 1 e 2, respectivamente.

3, 5, 6-Trimetoxi-[2”, 3": 7, 8]-furanoflavona (3). Cristais amarelos. p.f. $157,4-159,2{ }^{\circ} \mathrm{C}$, IV $(\mathrm{KBr}), \mathrm{cm}^{-1}$ : 2934; 2840; 1633; 1479; $1447 ; 1345 ; 1230 ; 1174 ; 1230 ; 1174 ; 1065$. EM (Int. rel.) $\mathrm{m} / z: 352$ ([M]+., 100), 337 (86), 321 (28), 308 (22), 205 (14), 177 (28), 147 (14), 105 (31), 77 (28). Os dados de $\mathrm{RMN}{ }^{13} \mathrm{C} \mathrm{e}{ }^{1} \mathrm{H}\left(\mathrm{CDCl}_{3}\right)^{11}$ estão descritos nas Tabelas 1 e 2, respectivamente.

2, 4', 4, 5-Tetrametoxi-[2", 3":7, 8]-furanodi-hidroaurona (4). Resina amarela. $[\alpha]_{\mathrm{D}}^{20}+96^{\circ}\left(0,004, \mathrm{CHCl}_{3}\right), \mathrm{IV}(\mathrm{KBr}), \mathrm{cm}^{-1}: 1717$, 1609, 1505, 1342, 1249, 1155, 1078. EM (Int. rel.) 384 ([M]+., 61), 353 (7), 293 (12), 263 (100), 248 (46), 220 (62), 205 (48), 177 (33), 121 (58), 77 (61). Os dados de $\mathrm{RMN}{ }^{13} \mathrm{Ce} \mathrm{e}^{1} \mathrm{H}\left(\mathrm{CDCl}_{3}\right)^{4}$ estão descritos nas Tabelas 1 e 2 , respectivamente.

3, 4', 5, 6-Tetrametoxi-[2", 3":7, 8]-furanoflavona (5). Cristais amarelos. p.f. $154,0-156,3{ }^{\circ} \mathrm{C}$, IV (KBr), $\mathrm{cm}^{-1}$ : 2936, 2842, 1617 , 1609, 1505, 1470, 1342; 1249; 1155; 1078. EM (Int. rel.) m/z: 382 ([M] $\left.]^{+}, 85\right), 367$ (100), 345 (65), 307 (17), 278 (11), 205 (20), 177 (48), 135 (21), 77 (20). Os dados de $\mathrm{RMN}^{13} \mathrm{C}$ e ${ }^{1} \mathrm{H}$ estão descritos nas Tabelas 1 e 2 , respectivamente.

(2R,3S,4S)-3, 4, 5, 6-Tetrametoxi-[2", 3":7, 8]-furanoflavana (6). Resina amarela. $[\alpha]_{\mathrm{D}}^{20}-1^{\circ}\left(0,004, \mathrm{CHCl}_{3}\right), \mathrm{IV}(\mathrm{KBr}), \mathrm{cm}^{-1}$ : 2937, 2831, 1680, 1622, 1483, 1130, 1062. EM (Int. rel.) $\mathrm{m} / \mathrm{z}: 370$ ([M] $\left.]^{+}, 17\right), 339$ (2), 236 (30), 221 (44), 193 (11), 134 (28), 91 (72). Os dados de $\mathrm{RMN}{ }^{13} \mathrm{C}$ e ${ }^{1} \mathrm{H}\left(\mathrm{CDCl}_{3}\right)^{3,12}$ estão descritos nas Tabelas 1 e 2 , respectivamente.

3', 4'-Metilenodioxi-5, 6-dimetoxi-[2",3":7,8]-furanoflavona (7). Sólido amorfo amarelo. p.f. 222,2-226, ${ }^{\circ} \mathrm{C}$, IV (KBr) $\mathrm{cm}^{-1}: 1646$; 1479; 1449; 1378; 1336 1257; 1130; 1068; 1028. EM (Int. rel.) m/z: 366 ([M] $\left.{ }^{+}, 71\right), 351$ (100), 337 (35), 322 (57), 295 914), 205 (18), 177 (48), 161 (28), 145 (17), 119 (10). Os dados de RMN ${ }^{13} \mathrm{C} \mathrm{e}{ }^{1} \mathrm{H}$ $\left(\mathrm{CDCl}_{3}\right)^{11}$ estão descritos nas Tabelas 1 e 2 , respectivamente.<smiles>COc1c2c(c(OC)c3occc13)O[C@@H](c1ccccc1)[C@H](OC)[C@H]2OC</smiles>

1<smiles>C=C[C@]1(C)C=Cc2c(c(OC)cc3c(=O)c(OC)c(-c4ccccc4)oc23)O1</smiles>

2<smiles>COc1c(-c2ccccc2)oc2c(c(OC)c(OC)c3occc32)c1=O</smiles>

3<smiles></smiles>

5<smiles>COc1c(OC)c2c(=O)cc(-c3ccc4c(c3)OCO4)oc2c2ccoc12</smiles>

7<smiles>COc1ccc(CC2(OC)Oc3c(c(OC)c(OC)c4occc34)C2=O)cc1</smiles>

4<smiles>COc1c2c(c3ccoc3c1OC)OC[C@H](c1ccccc1)[C@@H]2OC</smiles>

6
Figura 1. Estruturas dos flavonoides isolados de L. campestris 


\section{RESULTADOS E DISCUSSÃO}

O fracionamento cromatográfico dos extratos hexânico e etanólico das cascas das raízes de L campestris culminou no isolamento de sete flavonoides, cujas estruturas foram elucidadas por métodos espectroscópicos, em particular $\mathrm{RMN}{ }^{1} \mathrm{H}$ e ${ }^{13} \mathrm{C}, 1 \mathrm{D}$ e $2 \mathrm{D}$. Dos flavonoides isolados, 6 já haviam sidos relatados na literatura: $(2 S, 3 R, 4 S)-3,4,5,8$ tetrametoxi-[2",3":6,7]-furanoflavana (1), 3,6-dimetoxi-2",2"-dimetilcromeno-[2",3":7,8]-flavona (2), 3,5,6-trimetoxi-[2",3":7,8]-furanoflavona (3), 2,4',4,5-tetrametoxi-[2",3":6,7]-furanodi-hidroaurona (4), $(2 R, 3 S, 4 S)$-3,4,5,6-tetrametoxi-[2",3":7,8]-furanoflavana (6) e 3',4'-metilenodioxi-5,6-dimetoxi-[2",3":7,8]-furanoflavona (7), Figura 1. As estruturas das furanoflavanas $\mathbf{1}$ e $\mathbf{6}$ foram diferenciadas com base em seus dados de RMN e comparação com a literatura, considerando especialmente os deslocamentos químicos dos carbonos C-6 $(\mathbf{1}, 114,0 ; \mathbf{6}, 133,6)$ e C-8 $(\mathbf{1}, 126,7 ; \mathbf{6}, \mathbf{1 1 4}, 4)$. Adicionalmente, o experimento HMBC de $\mathbf{6}$ mostrou claramente a correlação entre o sinal de hidrogênio em $\delta 6,84$ (d, $J=2,1 \mathrm{~Hz}, \mathrm{H}-3$ ") com o sinal de carbono em $\delta 143,9$ (C-9) e também do sinal em $\delta$ 4,08 (s, 6-OMe) com o sinal em $\delta 133,6$ (C-6). Vale mencionar que, de acordo com o valor da rotação ótica, o composto 1 refere-se ao enantiômero (-)-3,4,5,8-tetrametoxi-[2",3":6,7]-furanoflavana. Os dados de RMN ${ }^{13} \mathrm{C}$ e ${ }^{1} \mathrm{H}$ dos compostos encontram-se descritos nas Tabelas 1 e 2, respectivamente.

Tabela 1. Deslocamentos químicos de $\mathrm{RMN}{ }^{13} \mathrm{C}\left(\mathrm{CDCl}_{3}\right)$ dos compostos 1 - 7

\begin{tabular}{|c|c|c|c|c|c|c|c|}
\hline No. & 1 & 2 & 3 & 4 & 5 & 6 & 7 \\
\hline 2 & 80,7 & 154,6 & 153,6 & 109,7 & 153,6 & 79,6 & 162,0 \\
\hline 3 & 83,1 & 141,3 & 141,0 & 40,7 & 140,2 & 82,1 & 106,6 \\
\hline 4 & 74,7 & 174,4 & 175,3 & 195,4 & 175,1 & 74,9 & 178,7 \\
\hline 5 & 147,5 & 104,5 & 147,5 & 147,0 & 147,4 & 148,8 & 147,6 \\
\hline 6 & 114,0 & 147,2 & 130,4 & 125,1 & 130,3 & 133,6 & 130,5 \\
\hline 7 & 148,7 & 146,6 & 149,3 & 155,3 & 149,1 & 148,5 & 149,6 \\
\hline 8 & 126,7 & 110,4 & 119,7 & 113,7 & 119,6 & 114,4 & 120,1 \\
\hline 9 & 145,2 & 147,5 & 146,3 & 144,5 & 146,1 & 143,9 & 146,9 \\
\hline 10 & 111,3 & 117,5 & 114,7 & 124,0 & 114,2 & 110,8 & 114,9 \\
\hline $1^{\prime}$ & 139,0 & 131,5 & 131,2 & 125,3 & 145,7 & 138,6 & 125,8 \\
\hline $2^{\prime}$ & 126,7 & 128,4 & 128,5 & 131,8 & 130,2 & 126,7 & 105,6 \\
\hline $3^{\prime}$ & 128,4 & 128,7 & 128,7 & 113,6 & 114,6 & 128,3 & 148,8 \\
\hline $4^{\prime}$ & 127,9 & 130,5 & 130,7 & 158,7 & 161,6 & 127,9 & 151,2 \\
\hline 5, & 128,4 & 128,7 & 128,5 & 113,6 & 114,6 & 128,3 & 109,1 \\
\hline 6 & 126,7 & 128,4 & 128,7 & 131,8 & 130,2 & 126,7 & 122,1 \\
\hline $1 "$ & - & 78,3 & - & - & - & - & - \\
\hline $2 "$ & 143,7 & 131,5 & 145,8 & 144,5 & 145,7 & 143,2 & 146,0 \\
\hline $3 "$ & 105,0 & 115,5 & 105,3 & 106,3 & 105,2 & 104,8 & 105,5 \\
\hline $2, "$ & - & - & - & - & - & - & 102,2 \\
\hline 2-OMe & - & - & - & 52,3 & - & - & - \\
\hline 3-OMe & 58,5 & 60,3 & 60,2 & - & 60,0 & 58,7 & - \\
\hline 4-OMe & 56,9 & - & - & 61,4 & - & 57,1 & - \\
\hline 5-OMe & 60,7 & - & 61,7 & 61,2 & 62,6 & 61,1 & 62,7 \\
\hline 6-OMe & - & 56,5 & 62,6 & - & 61,8 & 61,7 & 61,8 \\
\hline 8-OMe & 61,5 & - & - & - & & - & - \\
\hline 4'-OMe & - & - & - & 55,3 & 55,5 & - & - \\
\hline 2"-Me & - & 28,1 & - & - & & - & - \\
\hline
\end{tabular}

O composto 5 foi isolado na forma de cristais amarelos com ponto de fusão $154,0-156,3{ }^{\circ} \mathrm{C}$. Seu espectro no IV apresentou uma absorção em $1717 \mathrm{~cm}^{-1}$ correspondente à deformação axial de ligação $\mathrm{C}=\mathrm{O}$ e absorções em $1609 \mathrm{a} 1470 \mathrm{~cm}^{-1}$ referentes à deformação axial de ligação $\mathrm{C}=\mathrm{C}$ de aromáticos.

$\mathrm{O}$ espectro de $\mathrm{RMN}{ }^{1} \mathrm{H}\left(500 \mathrm{MHz}, \mathrm{CDCl}_{3}\right)$ apresentou sinais correspondentes a um sistema AA'BB'em $\delta 8,20$ (d, $J=8,9 \mathrm{~Hz}$, H-2'/H-6') e 7,04 (d, $J=8,9$ Hz, H-3'/H-5'), compatível com acoplamento orto em um anel aromático para-substituído; sinais em 7,62 (d, $\left.J=2,1 \mathrm{~Hz}, \mathrm{H}-2^{\prime \prime}\right)$ e 7,02 (d, $J=2,1 \mathrm{~Hz}, \mathrm{H}-3^{\prime \prime}$ ), relacionados a hidrogênios de um núcleo furânico $\alpha, \beta$-dissubstituído, além de singletos em $\delta 4,22 ; 4,11$ e 3,89 (2-OMe), típicos de grupos metoxila.

$\mathrm{O}$ espectro de $\mathrm{RMN}{ }^{13} \mathrm{C}-\mathrm{CPD}$ apresentou sinais correspondentes a 21 átomos de carbono, cujo padrão de hidrogenação foi determinado através do espectro DEPT 135. O sinal em $\delta 175,1$ (C-4) é compatível com uma carbonila conjugada de um núcleo flavonoídico. Os sinais em $\delta$ 130,2 e 114,6, correlacionados no espectro HMQC com os sinais de hidrogênio em $\delta$ 8,20 e 7,04, respectivamente, corroboraram o anel aromático para-substituído, enquanto os sinais em $\delta 145,7$ e 105,2, correlacionados aos sinais em $\delta$ 7,62 e 7,02, confirmaram um anel furânico. Os dados acima mencionados levaram a um flavonoide furânico tetra-metoxilado, cujo perfil estrutural poderia ser angular ou linear, como é peculiar aos flavonoides biossintetizados por plantas do gênero Lonchocarpus. No espectro HMBC, a correlação a três ligações, entre o sinal em $\delta 7,02$ (H-3'”) com o sinal de carbono em $\delta$ 146,1 (C-9) foi determinante para a localização do anel furânico em C-7 e C-8 (Figura 2). Com base nos dados espectroscópicos acima mencionados e sumarizados na Tabela 1 , determinou-se a estrutura 3,4',5,6-tetrametoxi (2"',3”:7,8:)- furanoflavona, a qual está sendo relatada pela primeira vez.

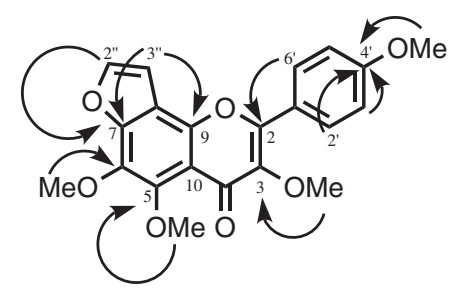

Figura 2. Principais correlações observadas no mapa de contornos HMBC de 5

\section{CONCLUSÕES}

Dos extratos hexânico e etanólico das cascas das raízes de $L$. campestris foram isolados sete flavonoides, incluindo quatro flavonas, duas flavanas e uma aurona. A exemplo da grande maioria dos flavonoides bioproduzidos por espécies de Lonchocarpus, todos os flavonoides isolados de L. campestris são metoxilados e apresentam anéis furânicos ou pirânicos como substituintes.

\section{MATERIAL SUPLEMENTAR}

As Figuras 1S a 6S estão disponíveis gratuitamente em http:// quimicanova.sbq.org.br, na forma de arquivo PDF.

\section{AGRADECIMENTOS}

Às instituições brasileiras de fomento à pesquisa CNPq e CAPES pelas bolsas de estudo e de pesquisador e à FUNCAP/PRONEX/ CNPq pelo apoio financeiro. 
Tabela 2. Deslocamentos químicos de $\mathrm{RMN}{ }^{1} \mathrm{H}\left(\mathrm{CDCl}_{3}\right)$ dos compostos $\mathbf{1}$ - 7

\begin{tabular}{|c|c|c|c|c|c|c|c|}
\hline No. & 1 & 2 & 3 & 4 & 5 & 6 & 7 \\
\hline 2 & $5,04(\mathrm{~d}, 6,5)$ & - & - & - & - & $5,17(\mathrm{~d}, 6,4)$ & - \\
\hline 3 & $3,92(\mathrm{dd}, 6,5 ; 4,2)$ & - & - & $\begin{array}{l}3,25(\mathrm{~d}, 13,9) \\
3,19(\mathrm{~d}, 13,9)\end{array}$ & - & $3,93(\mathrm{dd}, 6,4 ; 4,7)$ & $6,89(s)$ \\
\hline 4 & $4,80(\mathrm{~d}, 4,2)$ & - & - & - & - & $4,68(\mathrm{~d}, 4,7)$ & - \\
\hline 5 & - & 7,51 (m) & - & - & - & - & - \\
\hline $2^{\prime}$ & $7,48(\mathrm{~d}, 7,3)$ & $8,08(\mathrm{~d}, 7,3)$ & $8,20(\mathrm{dd}, 9,5 ; 1,6)$ & $7,16(\mathrm{~d}, 8,5)$ & $8,20(\mathrm{~d}, 8,9)$ & $7,45(\mathrm{~d}, 7,2)$ & $7,44(\mathrm{~d}, 1,7)$ \\
\hline $3^{\prime}$ & $7,38(\mathrm{t}, 7,1)$ & $7,51(\mathrm{~m})$ & $7,56(\mathrm{~m})$ & $6,88(\mathrm{~d}, 8,5)$ & $7,04(\mathrm{~d}, 8,9)$ & $7,37(\mathrm{t}, 7,1)$ & - \\
\hline 4 & $7,32(\mathrm{~d}, 7,3)$ & $7,51(\mathrm{~m})$ & $7,56(\mathrm{~m})$ & - & - & $7,32(\mathrm{~m})$ & - \\
\hline $5^{\prime}$ & $7,38(\mathrm{t}, 7,1)$ & $7,51(\mathrm{~m})$ & $7,56(\mathrm{~m})$ & $6,88(\mathrm{~d}, 8,5)$ & $7,04(\mathrm{~d}, 8,9)$ & $7,37(\mathrm{t}, 7,1)$ & $6,96(d, 8,2)$ \\
\hline 6 & $7,48(\mathrm{~d}, 7,3)$ & $8,08(\mathrm{~d}, 7,3)$ & $8,20(\mathrm{dd}, 9,5 ; 1,6)$ & $7,16(\mathrm{~d}, 8,5)$ & $8,20(\mathrm{~d}, 8,9)$ & $7,45(\mathrm{~d}, 7,2)$ & $7,60(\mathrm{dd}, 8,2 ; 1,7)$ \\
\hline $2 "$ & $7,51(\mathrm{~d}, 2,2)$ & $5,76(\mathrm{~d}, 10,0)$ & $7,63(\mathrm{~d}, 2,2)$ & $7,48(\mathrm{~d}, 2,0)$ & - & $7,52(\mathrm{~d}, 2,1)$ & $7,66(\mathrm{~d}, 2,0)$ \\
\hline $3 "$ & $6,87(\mathrm{~d}, 2,2)$ & $6,89(\mathrm{~d}, 10,0)$ & $7,04(\mathrm{~d}, 2,2)$ & $6,86(\mathrm{~d}, 2,0)$ & - & $6,84(\mathrm{~d}, 2,1)$ & $7,05(\mathrm{~d}, 2,0)$ \\
\hline $2^{\prime \prime \prime}$ & - & - & - & - & - & - & $6,09(\mathrm{~s})$ \\
\hline 3-OMe & $3,39(\mathrm{~s})$ & $3,88(\mathrm{~s})$ & $3,90(\mathrm{~s})$ & - & 3,89 (s) & $3,30(\mathrm{~s})$ & - \\
\hline 4-OMe & $3,36(s)$ & - & - & $4,18(\mathrm{~s})$ & - & $3,39(\mathrm{~s})$ & - \\
\hline 5-OMe & 4,07 (s) & - & $4,22(\mathrm{~s})$ & $4,07(\mathrm{~s})$ & $4,11(\mathrm{~s})$ & 3,99 (s) & $4,11(\mathrm{~s})$ \\
\hline 6-OMe & - & $3,96(\mathrm{~s})$ & $4,12(s)$ & - & $4,22(\mathrm{~s})$ & $4,08(\mathrm{~s})$ & 4,27 (s) \\
\hline 8-OMe & 4,05 (s) & - & - & - & - & - & - \\
\hline 4'-OMe & - & - & - & $3,69(\mathrm{~s})$ & $3,89(\mathrm{~s})$ & - & - \\
\hline $2 "$-Me & - & $1,55(\mathrm{~s})$ & - & - & - & - & - \\
\hline
\end{tabular}

\section{REFERENNCIAS}

1. Joly, A. B.; Botânica: Introdução a Taxonomia Vegetal, Ed. Nacional: São Paulo, 1993.

2. Polhill, R. M.; Raven, P. H.; Advances in Legume Systematics. Part 1, Royal Botanic Gardens: England, 1981.

3. Magalhães, A. F.; Tozzi, A. M. G. A.; Sales, B. H. L. N.; Magalhães, E. G.; Phytochemistry 1996, 42, 1459.

4. Lima, A. F.; Mileo, P. G. M.; Andrade-Neto, M.; Braz-Filho, R.; Silveira, E. R.; Pessoa, O. D. L. P.; Magn. Reson. Chem. 2009, 47, 165.

5. Magalhães, A. F.; Tozzi, A. M. G. A.; Magalhães, E. G.; Sannomiya, M.; Soriano, M. D. P. C.; Perez, M. A. F.; An. Acad. Bras. Ciênc. 2007, 79, 351.

6. Lawson, M. A.; Kauuadji, M.; Chulia, A. J.; Tetrahedron Lett. 2008, 49, 2407.
7. Campos, D. A.; Lima, A. F.; Ribeiro, S. R. L.; Silveira, E. R.; Pessoa, O. D. L.; Rao, V. S.; Santos, F. A.; J. Pharm. Pharmacol. 2008, 60, 391.

8. De Andrade Cunha, G. M.; Fontenele, J. B.; Nobre Junior, H. V.; De Sousa, F. C. M.; Silveira, E. R.; Nogueira, N. A. P.; Moraes, M. O.; Viana, G. S. B.; Costa-Lotufo, L. V.; Phytother. Res. 2003, 17, 155.

9. Fontenele, J. B.; Leal, L. K. A. M.; Ferreira, M. A. D.; Silveira, E. R.; Viana, G. S. B.; Pharm. Biol. 2005, 43, 726.

10. Khaomek, P.; Ichino, C.; Ishiyama, A.; Sekiguchi, H.; Namatame, M.; Ruangrungsi, N.; Saifah, E.; Kiyohara, H.; Otoguro, K.; Omura, S.; Yamada, H.; J. Nat. Med. 2008, 62, 217.

11. Nascimento, M. C.; Mors, W. B.; Phytochemistry 1981, 20, 147.

12. Almeida, J. R. G. S.; Silva, M. S.; Barbosa-Filho, J. M.; da-Cunha, E. F. L.; Braz-Filho, R.; Marques, A. S.; Zheng, C.; Ann. Magn. Reson. 2003, 1,33 . 


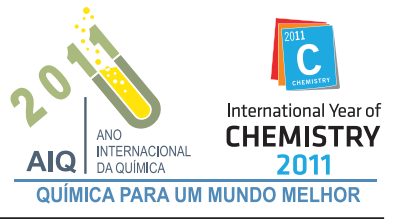

FLAVONOIDES DE Lonchocarpus campestris (Leguminosae)

Andreza Maria L. Pires, Edilberto R. Silveira e Otília Deusdênia L. Pessoa*

Departamento de Química Orgânica e Inorgânica, Centro de Ciências, Universidade Federal do Ceará, CP 12200, 60021-970 Fortaleza - CE, Brasil

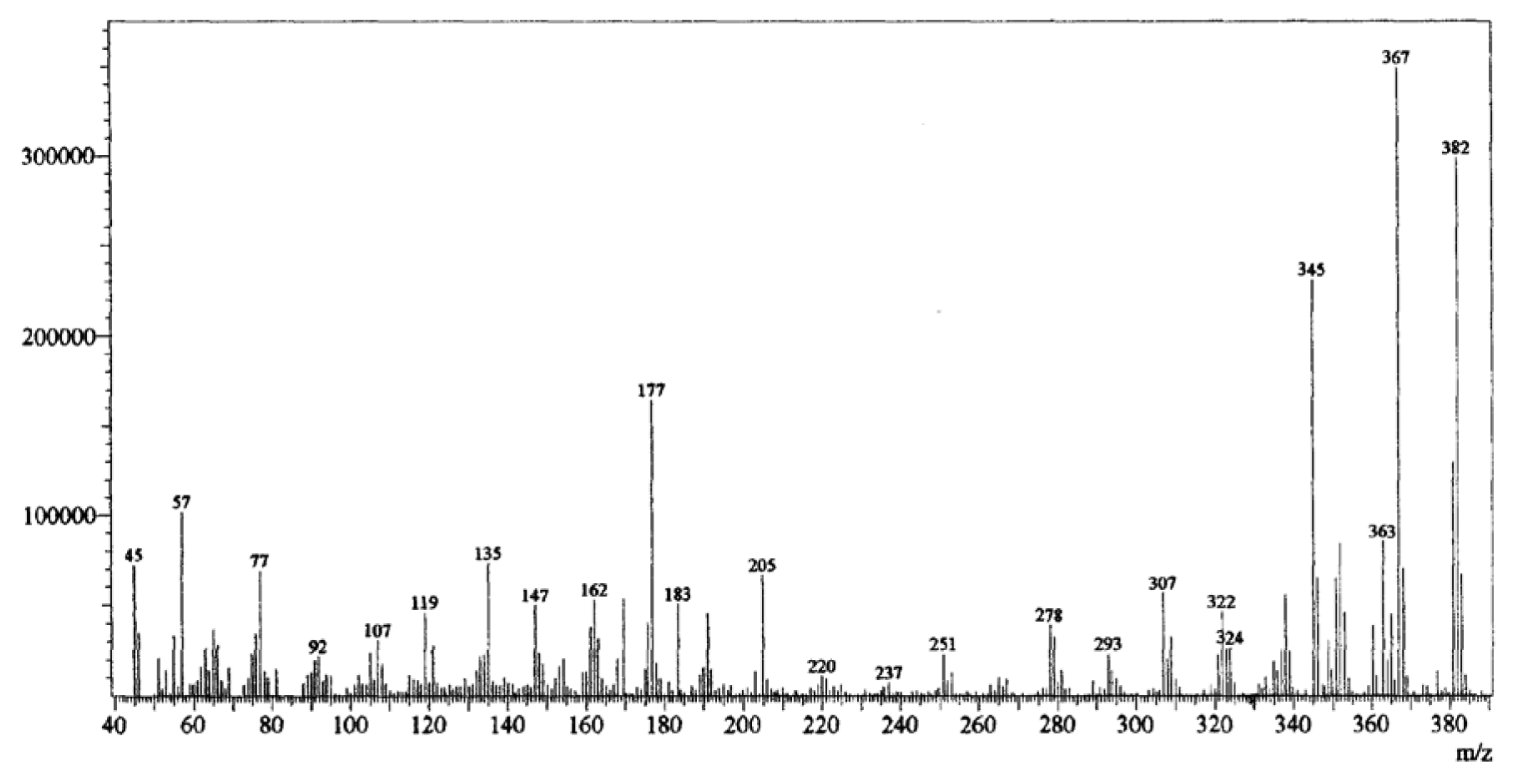

Figura 1S. Espectro de massas (70 eV) do composto 5 


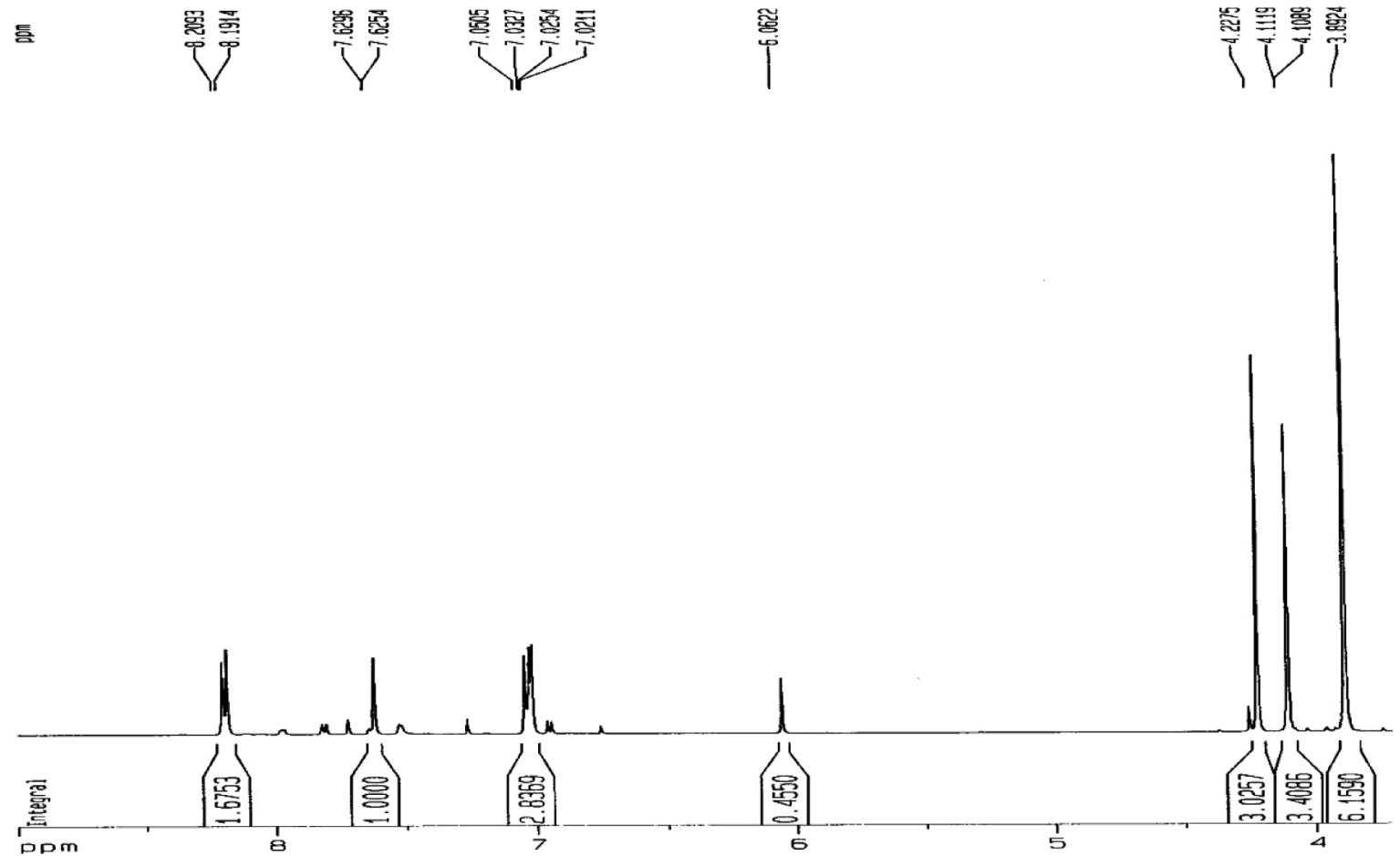

Figura $2 \mathrm{~S}$. Espectro de $\mathrm{RMN}^{l} \mathrm{H}\left(500 \mathrm{MHz}, \mathrm{CDCl}_{3}\right)$ do composto 5

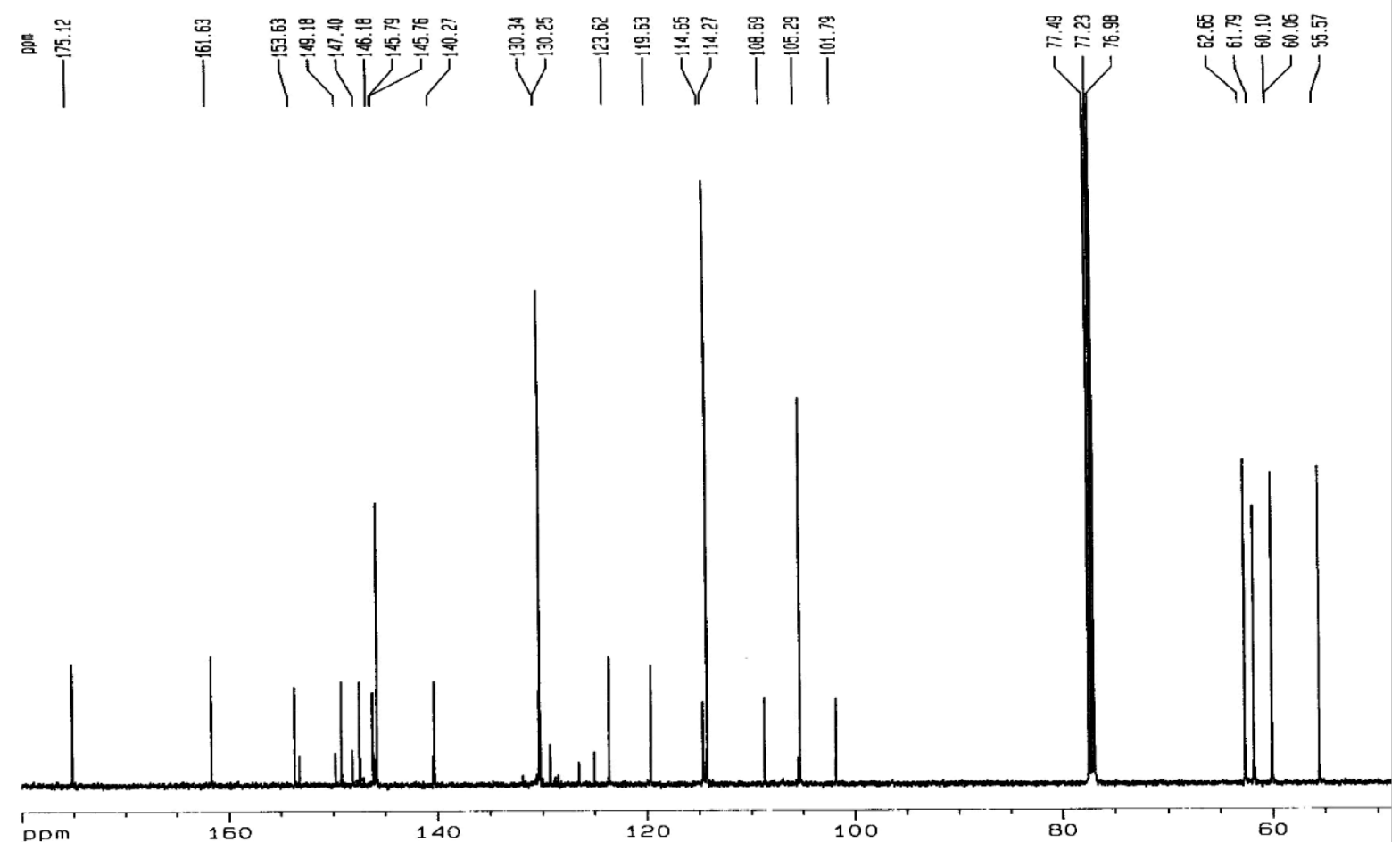

Figura 3S. Espectro de $\mathrm{RMN}^{13} \mathrm{C}\left(125 \mathrm{MHz}, \mathrm{CDCl}_{3}\right)$ do composto 5 
Vol. 34, No. 2

Flavonoides de Lonchocarpus campestris (Leguminosae)

S3

흥

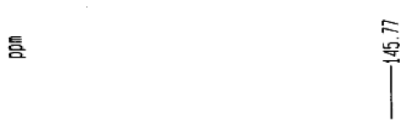

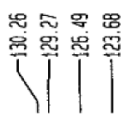

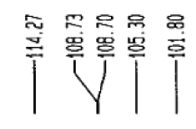

迢西三号 นึ่
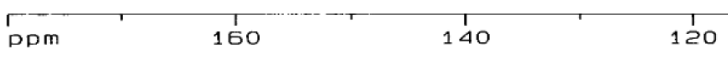

60

Figura 4S. Espectro de $\mathrm{RMN}^{13} \mathrm{C}-\mathrm{DEPT}\left(125 \mathrm{MHz}^{\mathrm{C}} \mathrm{CDCl}_{3}\right)$ do composto 5

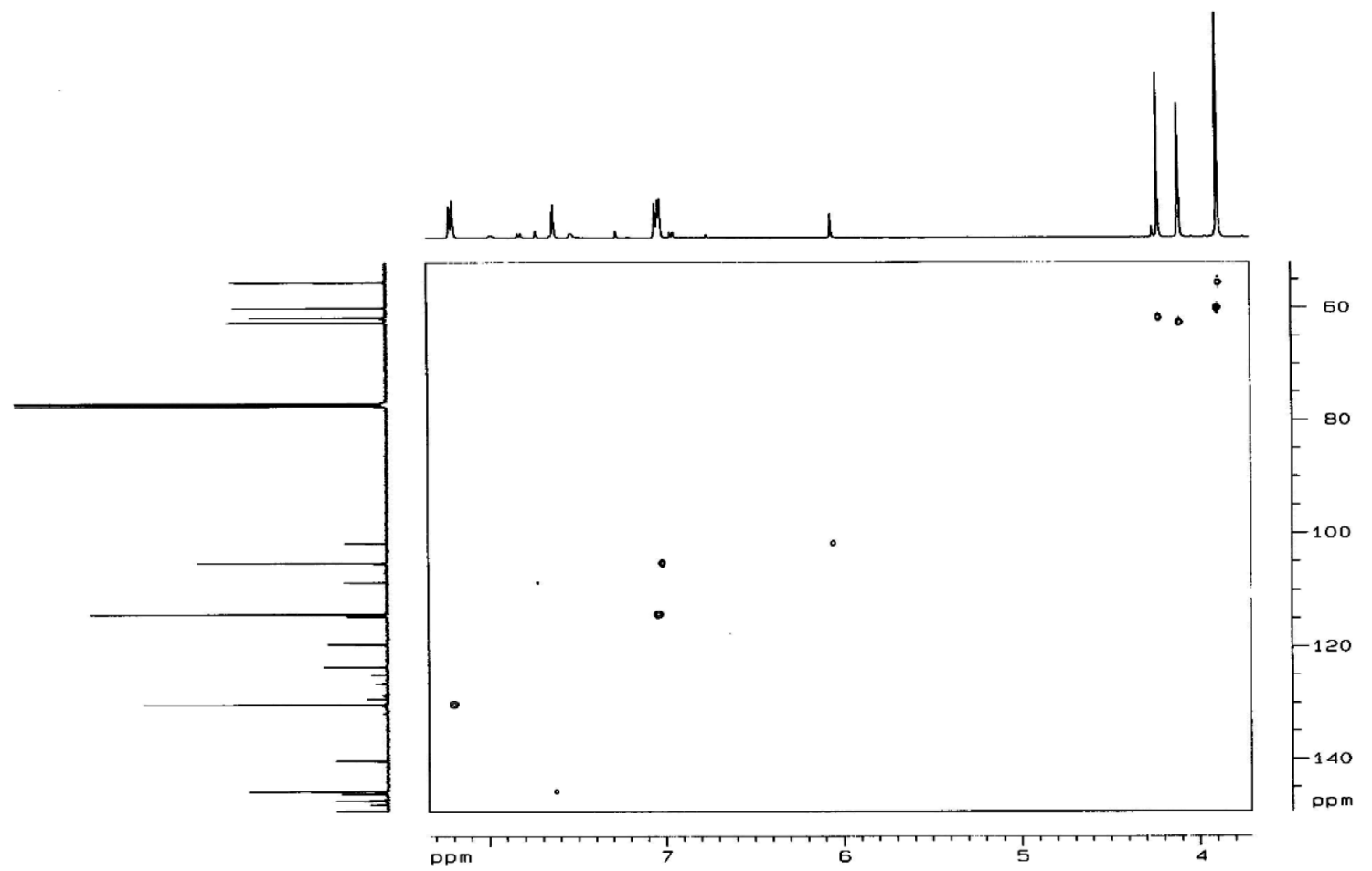

Figura 5S. Mapa de contornos $\mathrm{HMQC}\left(\mathrm{CDCl}_{3}\right)$ do composto 5 
S4

Pires et al.

Quim. Nova

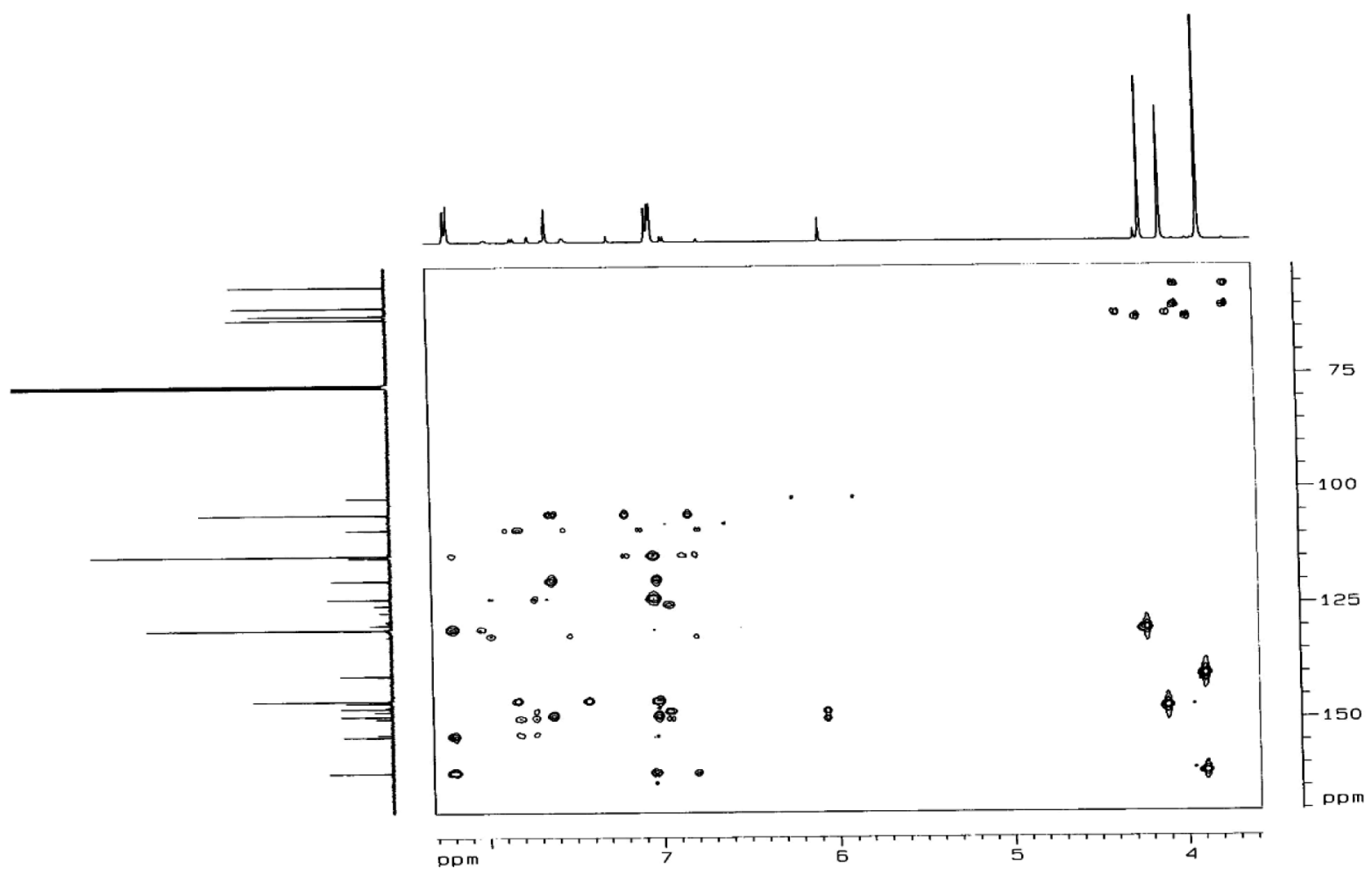

Figura 6S. Mapa de contornos $\mathrm{HMBC}\left(\mathrm{CDCl}_{3}\right)$ do composto 5 